\title{
Comportamento de um fluxo hiperpicnal gerado em simulação física: uma comparação entre fluxo confinado e não confinado
}

\author{
Daniel B. da SILVA ${ }^{1}$, Eduardo PUHL ${ }^{1}$, Rafael MANICA ${ }^{1}$, Adriano R.VIANA ${ }^{2}$ \& Ana L. de O. BORGES ${ }^{1}$ \\ 1.Núcleo de Estudos de Correntes de Densidade, Instituto de Pesquisas Hidráulicas, Universidade Federal do Rio Grande do Sul. Av. Bento \\ Gonçalves, 9500,CEP: 91.540-000, Porto Alegre, Brasil. E-mail: danibayer@bol.com.br, puhl.eduardo@gmail.com, manica@iph.ufrgs.br, \\ alborges@iph.ufrgs.br. \\ 2. Centro de Pesquisas e Desenvolvimento - CENPES, Petróleo Brasileiro S.A. Av. Horacio Macedo 950, Cidade Universitária, Ilha do Fundão, \\ CEP 21941-915, Rio de Janeiro, RJ, Brasil. E-mail: aviana@petrobras.com.br.
}

Recebido em 08/2012. Aceito para publicação em 05/2013.

Versão online publicada em xx/xx/20xx (www.pesquisasemgeociencias.ufrgs.br)

\begin{abstract}
Resumo- 0 estudo de fluxos hiperpicnais é extremamente importante dentro da indústria do petróleo, pois depósitos gerados por tais fluxos podem se tornar potenciais reservatórios de hidrocarbonetos. Uma das maneiras de entender esses processos sedimentares em ambiente marinho é através de experimentos em laboratórios (modelagem física), onde se controla os parâmetros envolvidos de maneira que reproduzam mais precisamente o fenômeno natural. Usando essa técnica de estudo, este trabalho visou comparar o comportamento de um fluxo hiperpicnal em duas situações distintas, ou seja, confinado em um canal subaquoso e fora do mesmo (não confinado). Buscou-se estabelecer as diferenças de hidrodinâmica e suas implicações no transporte e na deposição de sedimentos, durante um curto intervalo de tempo, de um experimento físico tridimensional de longa duração. Através da análise de imagens foi possível estabelecer correlações de velocidade, geometria e acelerações entre as duas situações do fluxo. Os resultados mostram que o fluxo, ao atingir a zona confinada, acelera espacialmente e localmente, modificando suas características geométricas (alongamento da região frontal) em relação ao fluxo que escoa fora da zona confinada. Nos ambientes naturais, esse tipo de fenômeno localizado e curto pode ocorrer por longas distâncias, com uma duração maior. Este confinamento com duração longa poderia proporcionar um aumento na velocidade do fluxo, propiciando a ocorrência maior de processos erosivos em relação a processos deposicionais.
\end{abstract}

Palavras-chave: fluxo hiperpicnal, confinamento de canal, modelagem física.

\begin{abstract}
HYPERPYCNAL FLOW BEHAVIOR GENERATED BY PHYSICAL SIMULATIONS: A COMPARISON BETWEEN CONFINED AND UNCONFINED FLows. The study of hyperpycnal flows (a type of sediment gravity flow) is extremely important in the petroleum industry, since deposits generated by such flows become potential hydrocarbon reservoirs. In order to understand these sedimentary processes in marine environment, laboratory experiments (physical modeling) with controlled input parameters are being performed more accurately to reproduce this natural complex phenomenon. Using this power tool, this work aims to compare the behavior of a hyperpycnal flow in two different situations, i.e., confined and unconfined flow in a subaqueous channel. We sought to establish the hydrodynamic differences and their implications in the transport and deposition of sediments during a short instant of time from a tridimensional physical experiment of long duration. Through the analysis of images was possible to establish correlations of velocity, acceleration and geometry between the two flow situations. The results show that the hyperpycnal flow generated at the beginning of the experiment accelerates both temporally and spatially as well as modifies its geometrical characteristics (elongation of the frontal region). In natural environments, such a locally and short phenomenon can occur through large distance and duration, this can afford an increase in flow velocity through the way, providing a greater occurrence of erosive process compared with depositional processes.
\end{abstract}

Key words: hyperpycnal flow, channel confined, physical modeling.

\section{Introdução}

Um dos principais processos de transferência de sedimentos para regiões profundas do oceano são os chamado fluxos gravitacionais de sedimentos (Middleton \& Hampton, 1976; Stow, 1994), os quais são classificados, segundo os mecanismos de ignição, principais forças atuantes, mecanismos de suporte dos grãos e seus depósitos característicos. Um tipo particular de fluxo gravitacional de sedimentos são as chamadas correntes hiperpicnais. 0 conceito de corrente hiperpicnal (hyperpycnal flow) foi introduzido por Bates (1953) como sendo um fluxo subaquoso de sedimento, deslocando-se pela base de uma coluna de água pela ação das forças gravitacionais diferenciais, as quais são devido à diferença de densidade entre a corrente e o meio (Fig. 
1). As correntes hiperpicnais estão fortemente ligadas às cheias fluviais, uma vez que elas fornecem à mistura de água e sedimentos, densidade necessária para que este evento ocorra e se mantenha ativo ao longo de um determinado tempo (Mulder \& Syvitski, 1995; Wheatcroft, 2000; Mulder et al., 2003).

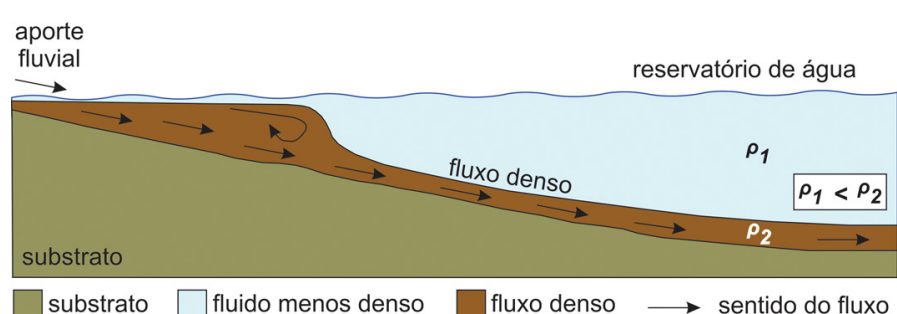

Figura 1. Modelo esquemático de uma corrente hiperpicnal, onde $\rho 1=$ densidade do fluido menos denso; $\rho 2=$ densidade do fluido mais denso. As setas indicam o sentido do fluxo denso.

Muitos estudos foram realizados para melhor compreender os processos de efeito/causa de correntes hiperpicnais (Kneller, 1995; Mulder \& Alexander, 2001; Gladstone \& Pritchard, 2010; Lamb et al., 2010). Enquanto Gladstone \& Pritchard (2010) e Lamb et al. (2010) demonstraram a formação e características dessas correntes em experimentos físicos, Mulder \& Alexander (2001) procuraram, com dados de campo, caracterizá-los e classificá-los baseando-se em suas propriedades físicas e mecanismos de suporte de grãos. Já Kneller (1995) associou o conceito hidrodinâmico da aceleração de uma partícula fluida, para descrever o comportamento dos fluxos gravitacionais através de variações temporais (permanência) e espaciais (uniformidade) da aceleração. Segundo o autor, o fluxo desacelerado (waning flow) se refere a um regime permanente, quando não há variações nas propriedades médias das partículas fluidas contidas em um volume de controle ao longo de um determinado tempo, já o fluxo acelerado (waxing flow) se refere a um regime não permanente, quando há variações dessas propriedades. Kneller (1995) também destacou que um escoamento que se torna mais rápido à jusante é denominado pelo autor de acumulativo (accumulative) e que se torna mais lento é denominado como depletivo (depletive). Esses estudos acompanham o crescente interesse da indústria do petróleo em elucidar questões provenientes da capacidade de formação de reservatórios de hidrocarbonetos estarem associados a este tipo de fluxo.

Apesar da grande relevância econômica, o que se conhece dos fluxos gravitacionais de sedimentos em ambiente marinho foi obtido, em sua maioria, por métodos indiretos, e.g. sísmicas, observação de testemunho a pistão (piston cores) e por analogia de sistemas deposicionais em terra. Por outro lado, a observação e análise in loco destes processos ainda são dificultadas pelo seu caráter imprevisível e pelas grandes dimensões desse fenômeno, prejudicando o entendimento global desses processos no ambiente natural.

Um dos métodos empregados na análise de tais processos, o qual vem contribuindo para o avanço na compreensão destes fluxos, é a modelagem física em laboratório, onde se procura estabelecer relações de semelhança com o sistema natural através da simulação destes fluxos com parâmetros controlados (causas) e relacionar posteriormente com os seus depósitos (efeitos). Com essa técnica de estudo, é possível uma minuciosa observação e quantificação dos processos envolvidos. Muitas vezes, a riqueza de detalhes obtidas nesses experimentos serve de base para a compreensão global do fenômeno, uma vez que as observações, coleta de dados, informações e verificação de fenômenos localizados são avaliados ao longo de todo o tempo que é reproduzido o processo físico no laboratório.

No Brasil, esse método de estudo vem sendo realizado pelo Necod (Núcleo de Estudos de Correntes de Densidade), no Instituto de Pesquisas Hidráulicas (IPH), Universidade Federal do Rio Grande do Sul (UFRGS), o qual desenvolve diversos experimentos envolvendo modelagem física de fluxos gravitacionais de sedimentos na tentativa de compreender as suas especificidades (e.g., Fabian, 2002; Del Rey, 2006; Ducker, 2007; Boffo, 2010; Manica, 2012).

Neste trabalho, foi avaliado um fenômeno, de curto intervalo de tempo, inserido em um experimento físico com uma duração maior, desenvolvido no Necod. 0 estudo buscou interpretar a diferença de comportamento hidrodinâmico de uma corrente hiperpicnal ao confinar-se em um canal subaquoso e suas implicações no transporte e na deposição de sedimento.

\section{Materiais e métodos}

Nesta seção serão descritos, primeiramente, o modelo físico utilizado e os ensaios previamente realizados e, posteriormente, as condições do ensaio e os métodos de obtenção dos dados analisados neste estudo. 0 fenômeno de curta duração avaliado (corrente hiperpicnal confinada) ocorreu na parte final de uma longa série de ensaios, portanto, sobre uma condição morfológica que já estava anteriormente estabelecida no modelo físico. Esses ensaios produziram uma morfologia deposicional relativa a um ambiente de plataforma / talude / bacia, o que possibilitou que fosse criado, manualmente, um canal com uma porção subaérea e outra subaquosa.

\subsection{Modelo físico}

O modelo físico utilizado nos experimentos foi um canal tridimensional de geometria simplificada (Manica, 2002), construído em alvenaria e com as seguintes dimensões internas: profundidade de $1,20 \mathrm{~m}$, comprimento de $13,85 \mathrm{~m}$, sendo que nos primeiros $6,85 \mathrm{~m}$ do comprimento, a largura é de $0,96 \mathrm{~m}$ e, nos 7,00 m finais, possui 4,66 m de largura (Fig. 2). 0 fundo do modelo é composto por três regiões distintas: plataforma, talude e bacia. Na figura 2 encontra-se um esquema em planta 
e um perfil A - B da configuração do fundo, mostrando suas dimensões e declividades em cada região. Um sistema auxiliar de alimentação de sedimentos composto por um reservatório com capacidade para 5000 l foi utilizado para preparar e armazenar a mistura de água e sedimento (composto por carvão) utilizada nos experimentos, além de um controlador automático de vazão.
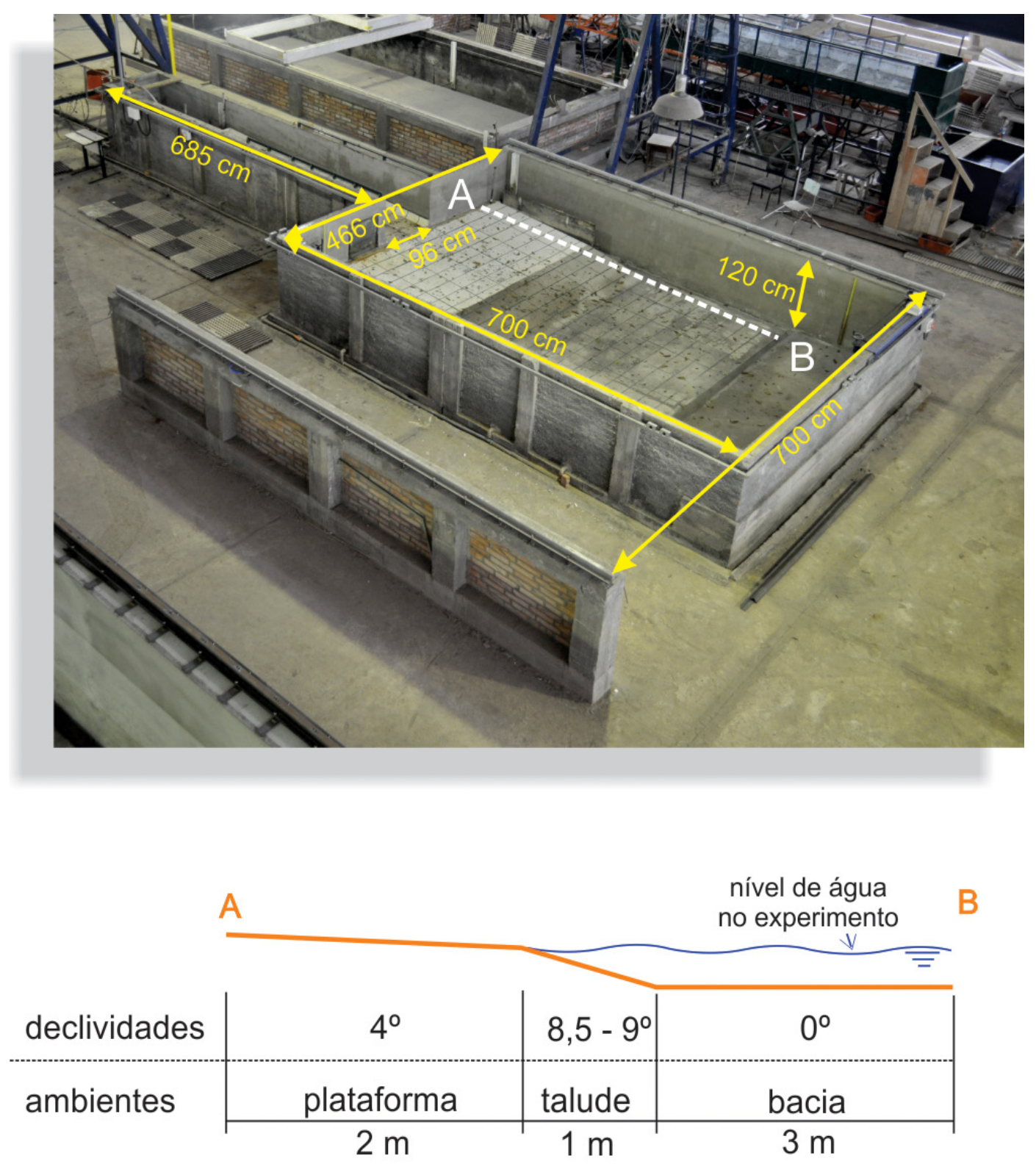

\section{Perfil A - B}

Figura 2. Tanque de experimentos utilizado neste trabalho e o perfil com medidas e declividades.

\subsection{Ensaios prévios}

Os ensaios de longa duração previamente realizados consistiram na injeção pontual de uma mistura de água e sedimentos, diluída na região canalizada do modelo (Fig. 2). Foram simuladas diversas variações de concentração de sedimentos, vazão de injeção e nível base do modelo (i.e. o nível estático de água dentro do modelo), resultando na formação do que representaria um delta fluvial na região da plataforma. A mistura era composta por água limpa (densidade de 0,99 ) e carvão mineral com diâmetro médio de $0,075 \mathrm{~mm}$ e densidade de 1,19 , tendo sido injetada durante 14 h e $40 \mathrm{~min}$.
Após o esvaziamento do tanque, sobre o depósito formado foram colocados grãos coloridos artificialmente com corante azul (areia com diâmetro médio de 0,25 $\mathrm{mm}$ ) para melhorar a visualização do experimento. Por decisão da equipe de simulação, ao final dessa série de ensaios, um canal de aproximadamente $10 \mathrm{~cm}$ de largura e com profundidade variável (média de $5 \mathrm{~cm}$ no delta e $0,5 \mathrm{~cm}$ no talude), foi escavado seguindo algumas feições já criadas naturalmente, desde a entrada da mistura (difusor) até um pouco à frente da base do talude (Fig. 3). Essa feição serviu de passagem da mistura para as regiões mais distais do modelo. 


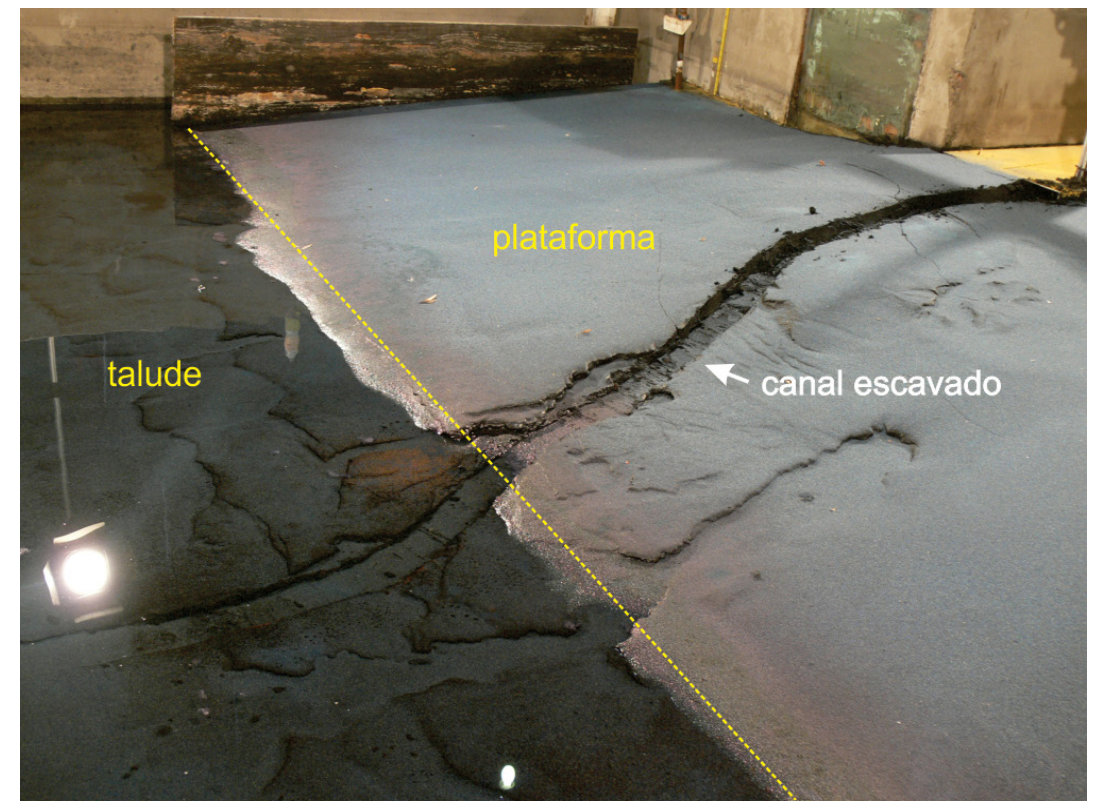

Figura 3. Depósito previamente formado indicando a posição do talude e plataforma, além do canal escavado e utilizado neste trabalho.

\subsection{Ensaio avaliado}

0 experimento em análise neste trabalho foi realizado com o difusor posicionado no topo da plataforma (Fig. 4). 0 nível de base do tanque se encontrava no topo do talude, totalizando cerca de $20 \mathrm{~cm}$ de fluido ambiente. A mistura de água e sedimentos possuía uma concentração de sedimentos de 8,8\% em volume, e era injetada com uma vazão de $4 \mathrm{l} \mathrm{min}^{-1}$. Este experimento durou um total de $1 \mathrm{~h}$ e $21 \mathrm{~min}$, no entanto o tempo de análise deste trabalho se restringiu ao momento inicial do experimento (aproximadamente os primeiros $30 \mathrm{~s}$ ), tempo que foi possível observar o processo e suficiente para analisar o comportamento do fluxo confinado e não confinado.

Durante o experimento, foram obtidas imagens com uma câmera fotográfica posicionada em uma plataforma acima do tanque, além de uma câmera de vídeo na lateral do mesmo (Fig. 4). Ambos os equipamentos serviram para detalhar a evolução da corrente hiperpicnal e avaliar seu comportamento hidrodinâmico.

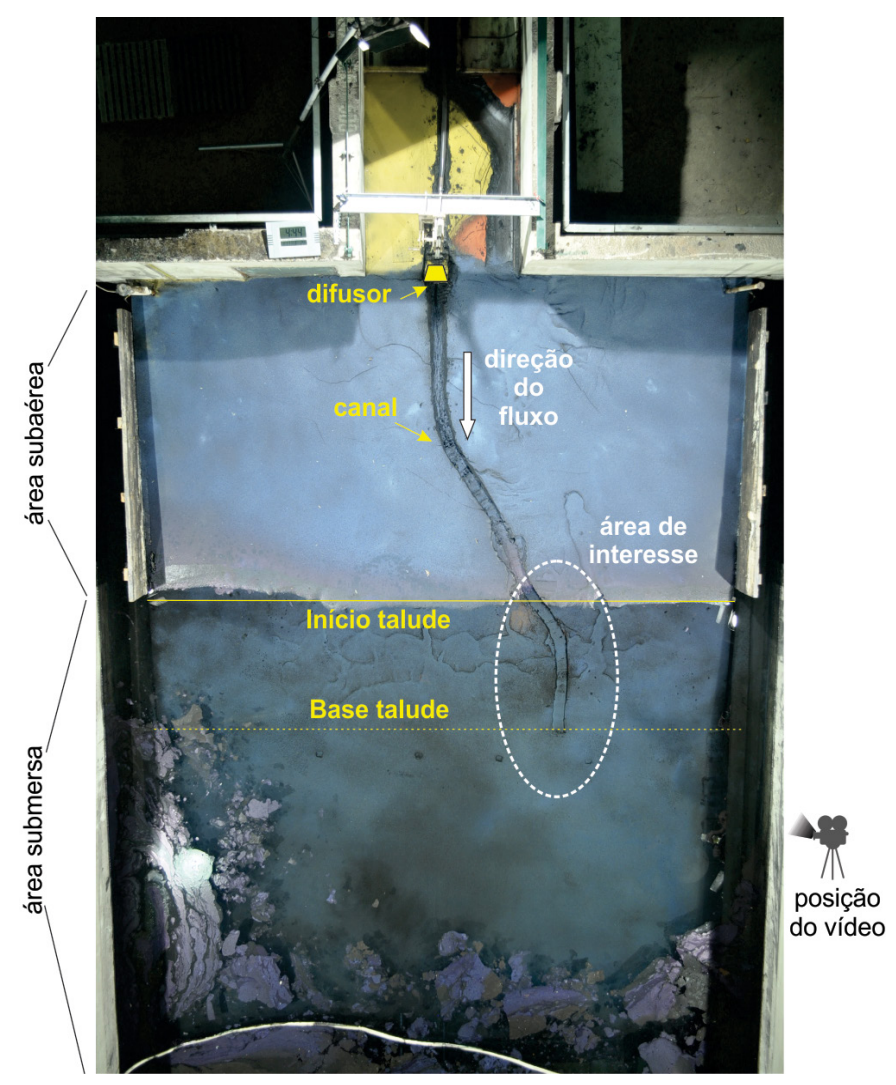

Figura 4. Tanque de experimentos indicando as principais feições do depósito e destacando a área de estudo deste trabalho (área tracejada). 


\section{Resultados}

As imagens obtidas pela câmera de vídeo (Fig. 5) foram organizadas em um mosaico sequencial (a cada segundo a partir do momento em que o fluxo chegou ao nível da água), com início no canto superior esquerdo, onde é possível visualizar a evolução da corrente hiperpicnal formada sobre o depósito prévio. Nota-se que, após $5 \mathrm{~s}$, parte da corrente hiperpicnal confinou-se no canal subaquático (destacado na figura 5), alterando sua geometria e formando uma frente de fluxo distinta do restante da corrente hiperpicnal. Esse fluxo confinado, por sua vez, acelera e ultrapassa o fluxo não confi- nado (visível à direita do fluxo confinado) entre $t=7 \mathrm{~s} \mathrm{e}$ $\mathrm{t}=8 \mathrm{~s}$, o qual não foi capaz de reverter esta situação até o final do trecho em análise. Entre os instantes $t=12$ $\mathrm{s}$ e $\mathrm{t}=13 \mathrm{~s}$, o fluxo confinado chegou ao final do canal previamente formado, ainda sendo possível distinguir uma região de fluxo mais a frente (cabeça da corrente) do restante do corpo da corrente. Já na parte final do ensaio ( $t>16 \mathrm{~s}$ ) a corrente hiperpicnal exibiu uma forma de fluxo homogeneizado, ou seja, não se distinguia a parte confinada da parte não confinada. Sendo assim, não foi possível visualizar se o canal condicionou o fluxo mesmo depois de seu término.

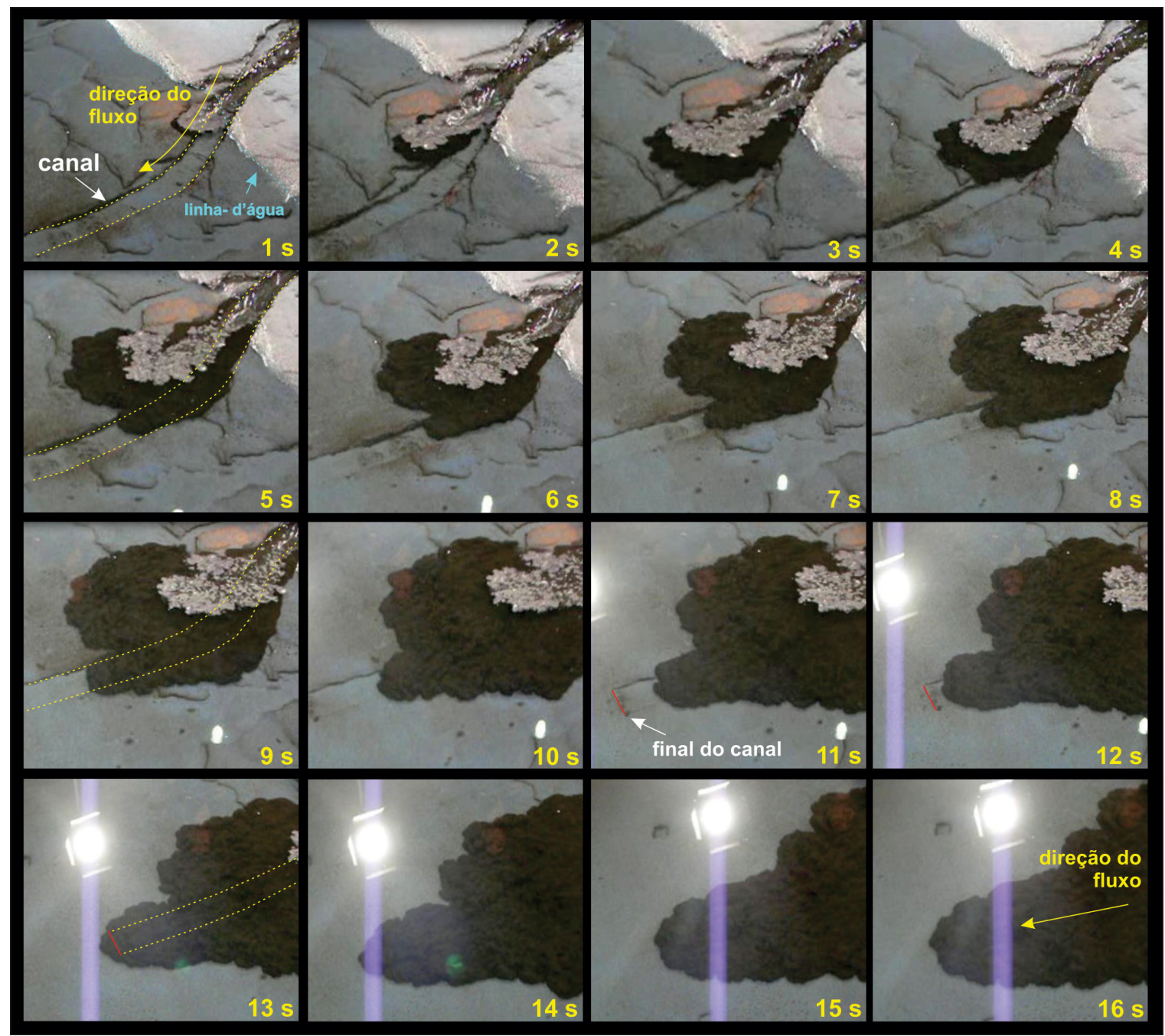

Figura 5. Fotos em sequência dos primeiros $16 \mathrm{~s}$ da corrente a partir do vídeo gravado lateralmente ao fluxo. 0 material de coloração mais clara associada a corrente, restrito a porção proximal do fluxo é a impureza contida no carvão. 0 canal está destacado por linhas tracejadas em $1 \mathrm{~s}, 5 \mathrm{~s}, 9 \mathrm{~s}$ e $13 \mathrm{~s}$.

Esse comportamento (Fig. 5) pode ser mais bem observado numa figura em planta, onde é mostrada sua evolução temporal (Fig. 6). A evolução da frente do fluxo (traçados na figura 6) demonstra a direção preferencial da corrente (sobre o canal formado previamente) exercendo influência no comportamento hidrodinâmico (representado pelo contorno geométrico mais alongado) ao contrário do restante da corrente (que flui sobre uma região não confinada), que apresentou velocidades menores. Para os momentos $\mathrm{t}=19 \mathrm{~s}$ e $\mathrm{t}=$
23 s, não foi possível realizar o contorno na frente da corrente por problema de resolução das imagens geradas pela câmera fotográfica.

A partir da visualização da evolução da frente da corrente hiperpicnal foi possível determinar a taxa de deslocamento dos fluxos (confinado e não confinado). No entanto, por não haver escala nas imagens, a dimensão horizontal do talude (1 $\mathrm{m}$ de comprimento) foi utilizada como escala geométrica. Com a utilização da dimensão do talude como escala e o tempo de cada 
imagem, foram calculadas as velocidades instantâneas (Fig. 7), tanto para o fluxo confinado quanto para o fluxo não confinado, desde o início do escoamento subaquático junto à linha-d'água $(\mathrm{t}=0 \mathrm{~s})$ até a junção da frente dos dois fluxos $(t \sim 18 \mathrm{~s})$, quando, aparentemente, o fluxo tornou-se homogêneo e já não era possível observar o canal. De acordo com o gráfico apresentado na figura 7, observa-se que houve uma velocidade ini- cial $\left(t=0\right.$ a 4 s) elevada $\left(25 \mathrm{~cm} \mathrm{~s}^{-1}\right)$ em relação ao restante da corrente, isto se deveu ao fato do escoamento ingressar em uma porção com maior declividade $(8,5$ - $9^{\circ}$ ), diminuindo a velocidade da frente da corrente posteriormente. Para melhor compreensão dos dados, a comparação dos fluxos será feita no período posterior a essa alta velocidade, a partir dos $6 \mathrm{~s}$.

A corrente hiperpicnal confinada se desenvolveu

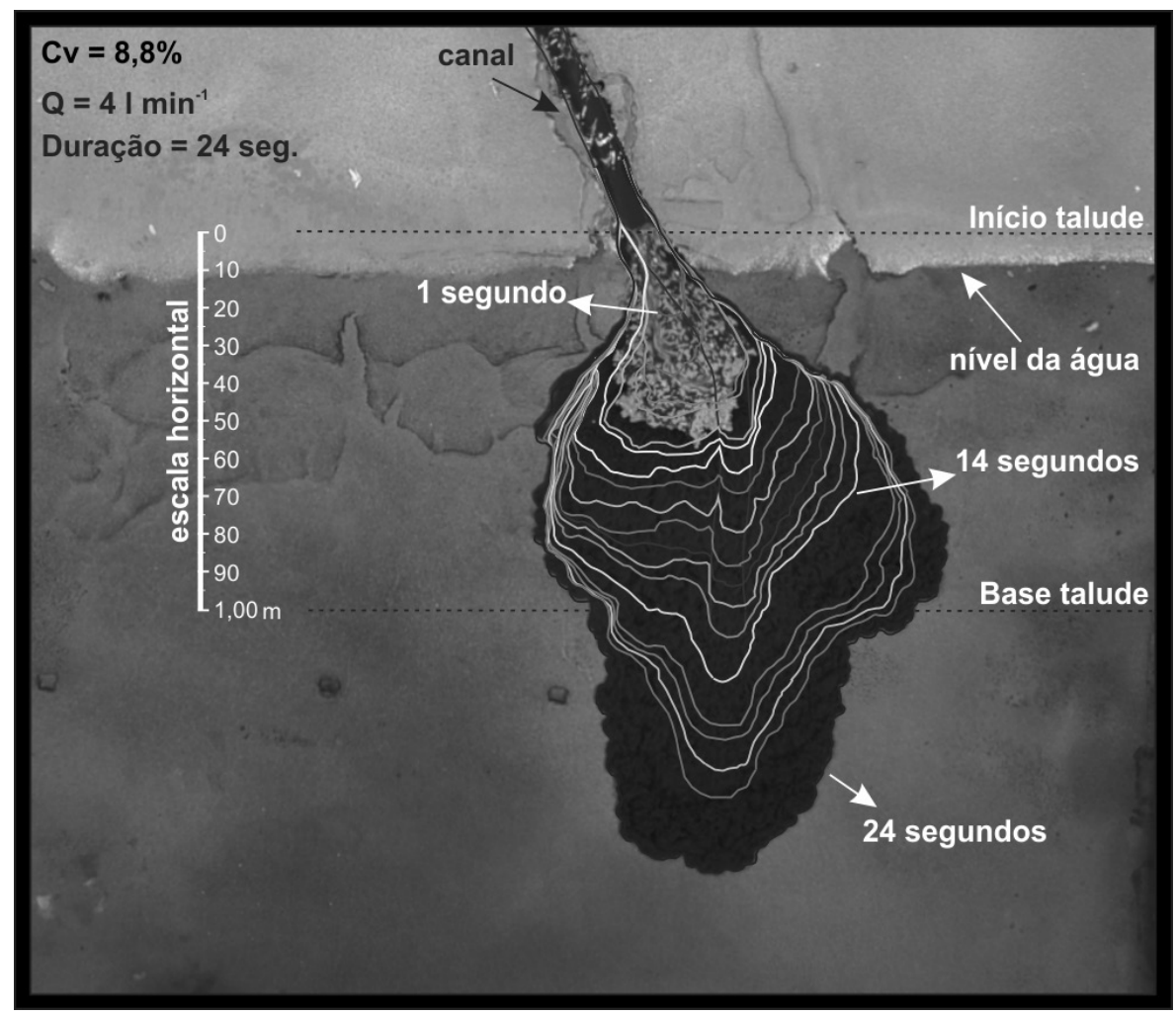

Figura 6. Esquema mostrando as linhas de evolução da frente da corrente hiperpicnal. Cada linha representa $1 \mathrm{~s}$ na evolução da corrente dentro desta região do tanque.

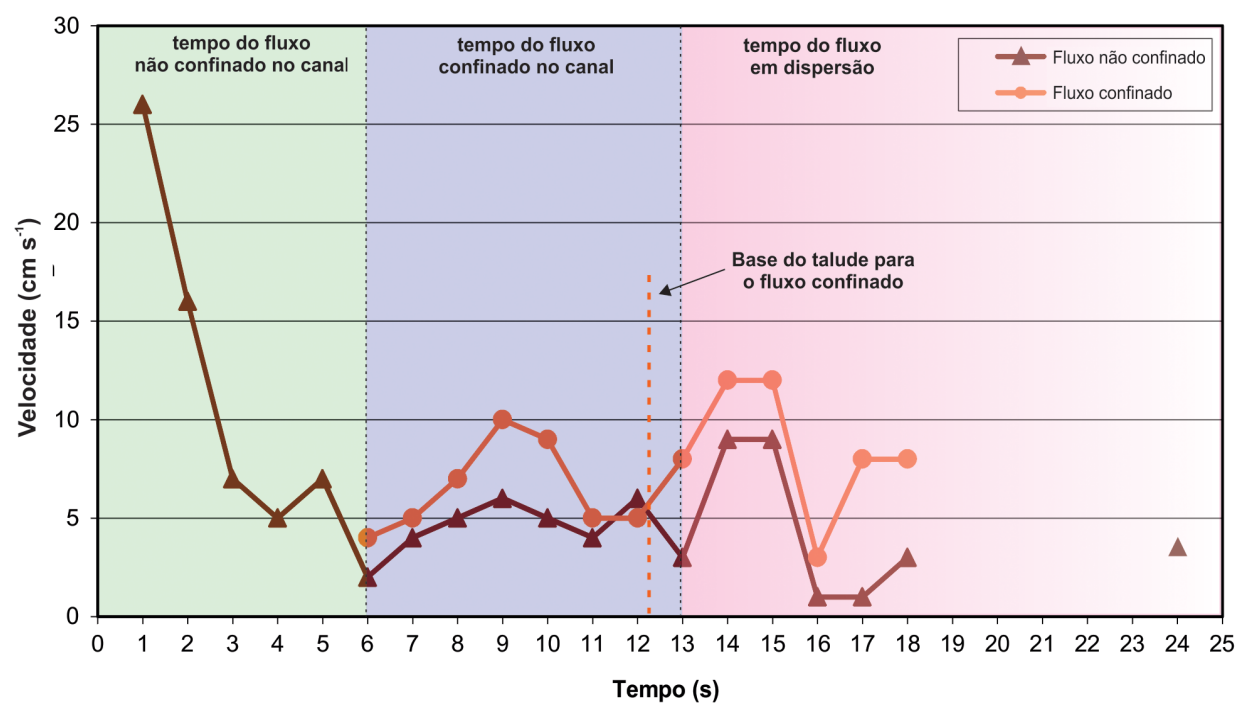

Figura 7. Gráfico das velocidades instantâneas dos fluxos confinado e não confinado ao longo do tempo. A área mais escura (intervalo de tempo $=6 \mathrm{~s}-13 \mathrm{~s}$ ) mostra o tempo em que o fluxo confinado esteve dentro do canal, já a linha tracejada indica o momento em que o fluxo confinado alcançou a base do talude.

a partir do momento em que ela escoou sobre o canal previamente formado $(t=5 \mathrm{~s})$, e permaneceu nessa condição até, aproximadamente, $\mathrm{t}=13 \mathrm{~s}$ (indicado na figura 7). Durante quase todo esse período de análise, o fluxo confinado sempre apresentou valores de velocidade instantâneas superiores ao fluxo que não estava escoando dentro do canal (fluxo não confinado), com exceção do momento em que a corrente hiperpicnal 
alcançou a base do talude, identificado pela linha tracejada $(\mathrm{t}=12-13 \mathrm{~s})$ indicada na figura 7. A transição do escoamento de uma declividade mais alta para uma mais baixa, conduz o escoamento a reduzir sua energia gravitacional (proporcional ao gradiente). No caso do experimento, foi possível observar a geração de um ressalto hidráulico nesta região, fenômeno no qual ocorre a "passagem do escoamento supercrítico para o subcrítico, com grande turbulência e perda de energia" (Baptista \& Coelho, 2003), ou seja, caracterizando uma desaceleração da corrente na região da quebra de declividade. Neste caso, o fluxo confinado dissipa parte da sua energia, ocasionando essa pequena diminuição de valores de velocidades instantâneas. Porém, após a passagem da quebra de declividade, ambos os fluxos alcançaram seus valores de velocidade máxima no período de análise. Este aumento de velocidade pode ter ocorrido devido a uma aceleração do fluxo no corpo da corrente, retornando ao seu estado de equilíbrio, interrompida pelo quebra de declividade.

A tabela 1 apresenta uma comparação de parâmetros cinemáticos entre o fluxo hiperpicnal confinado, o fluxo não confinado e o fluxo total, calculados para o perí- odo de comparação compreendido entre $t=5 \mathrm{~s} \mathrm{e} t=18 \mathrm{~s}$.

\begin{tabular}{lccccc}
\hline & $\begin{array}{c}\text { Distância } \\
\text { percorrida } \\
(\mathrm{cm})\end{array}$ & $\begin{array}{c}\text { Vel. } \\
\text { média } \\
(\mathrm{cm} / \mathrm{s})\end{array}$ & $\begin{array}{c}\text { Vel. } \\
\text { máxima } \\
(\mathrm{cm} / \mathrm{s})\end{array}$ & $\begin{array}{c}\text { Vel. } \\
\text { mínima } \\
(\mathrm{cm} / \mathrm{s})\end{array}$ & $\begin{array}{c}\text { Tempo } \\
(\mathrm{s})\end{array}$ \\
\hline $\begin{array}{l}\text { Fluxo confinado } \\
\text { Fluxo não }\end{array}$ & 96 & 7,38 & 12 & 3 & 13 \\
$\begin{array}{l}\text { confinado } \\
\text { Fluxo total }\end{array}$ & 58 & 4,46 & 9 & 1 & \\
\hline
\end{tabular}

Tabela 1. Dados de comparação relativa a um intervalo de tempo de $13 \mathrm{~s}$ entre os fluxos confinado e não confinado. 0 fluxo total foi assim chamado, por não distinguir ambos os fluxos, considerando-o como um só.

Pela análise dos valores apresentados na tabela 1 , verifica-se que o fluxo confinado teve uma velocidade média superior ao fluxo não confinado no intervalo de tempo em que eram distintos. Além de apresentar velocidades média, máxima e mínima maiores, a distância percorrida pelo fluxo confinado é 1,65 vezes maior (Fig. 8) que o fluxo não confinado para este intervalo de tempo.

\section{Fluxo não confinado}

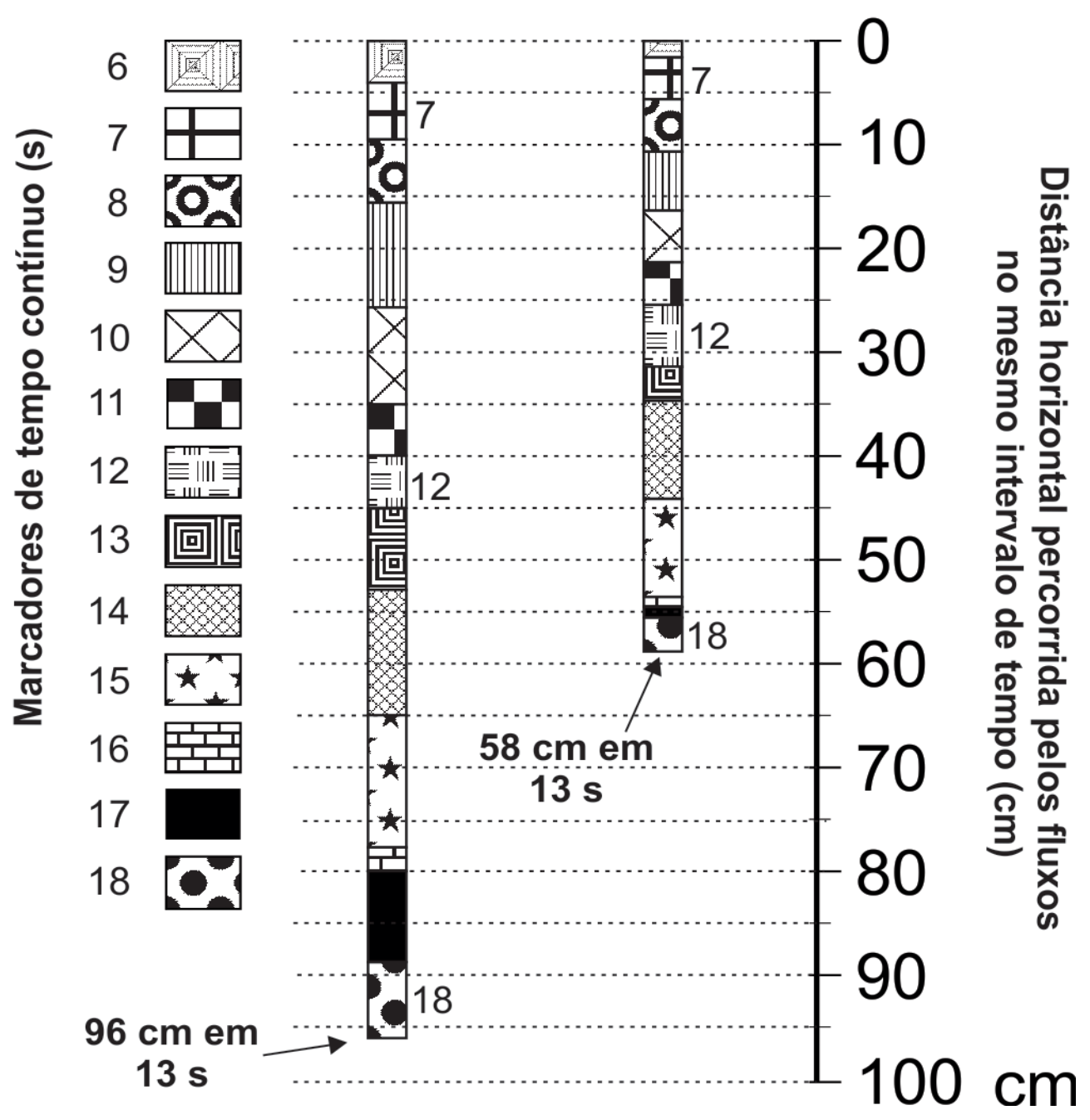

Figura 8. Esquema que destaca a distância horizontal percorrida por cada fluxo no mesmo intervalo de $13 \mathrm{~s}$. 
A Fig. 9 apresenta a análise da variação da aceleração instantânea dos fluxos confinado e não confinado ao longo dos $23 \mathrm{~s}$ de simulação. Em ambos os fluxos, nota-se um comportamento semelhante de acelerações e desacelerações, a qual possui uma frequência na ordem de $0,5 \mathrm{~Hz}$. Tal comportamento já foi atestado por outros autores (Simpson \& Britter, 1979; Fabian, 2002) e está fortemente associado a fenômenos presentes na interface superior do fluxo (mistura e desprendimentos de vórtices do tipo Kelvin-Helmholtz) e ainda, na geração de ondas internas provocadas por estratificação de material sedimentar ao longo do seu escoamento (Del Rey, 2006; Felix \& Peakall, 2006; Manica, 2009) na interface superior da corrente.
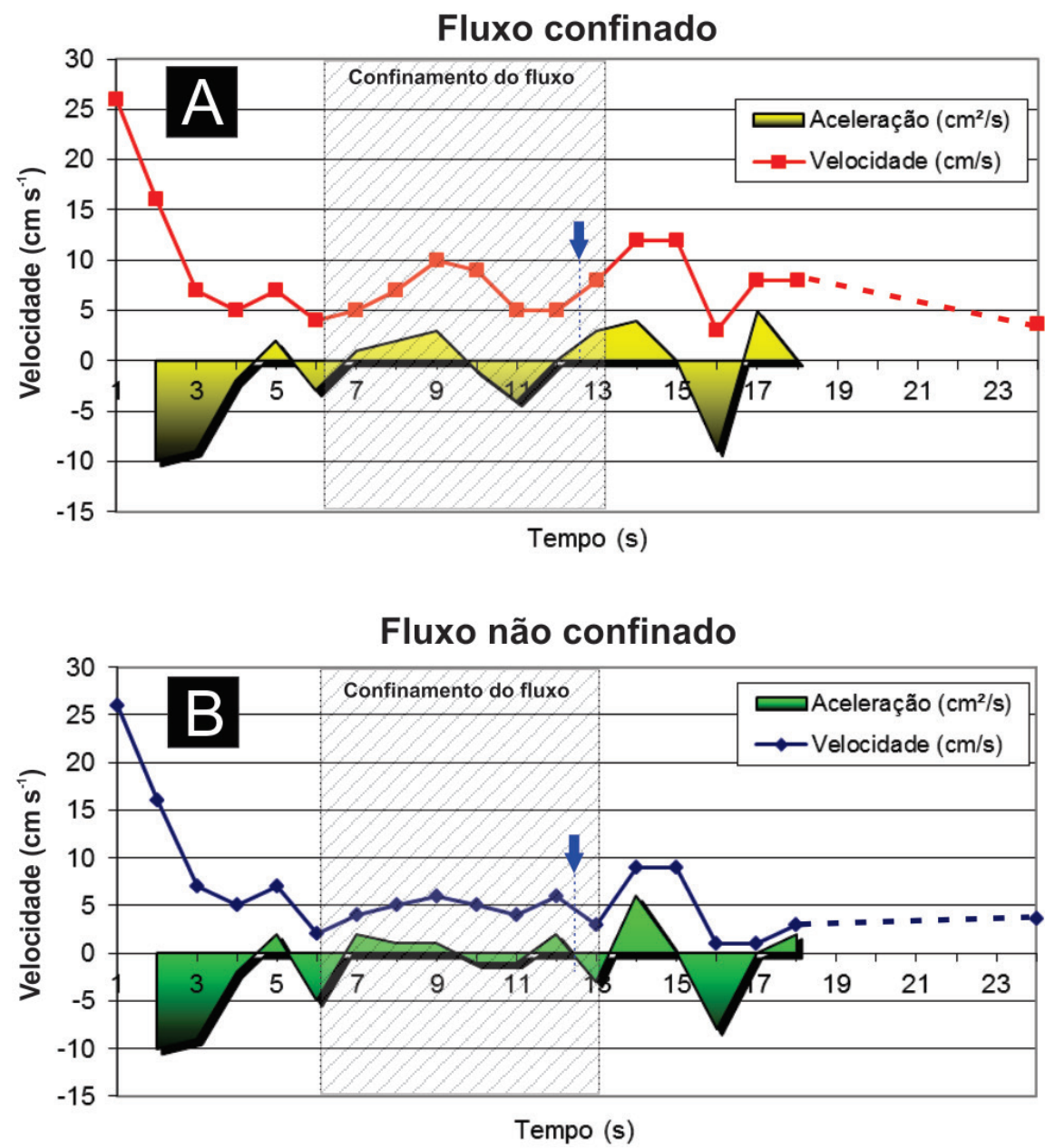

Figura 9. Gráficos das velocidades e acelerações instantâneas do fluxo confinado (A) e não confinado (B) ao longo do tempo. A área retangular com linhas diagonais indica o tempo em que o fluxo confinado esteve dentro do canal, já a seta indica o momento em que o fluxo confinado alcançou a base do talude.

\section{Discussão dos resultados}

O fenômeno físico reproduzido neste trabalho, através da modelagem física de fluxos gravitacionais de sedimentos, buscou criar uma situação semelhante a um rio aportando sua carga fluvial diretamente no talude continental do corpo receptor, representando um cenário geológico de rebaixamento do nível do mar com exposição da plataforma continental ou simplesmente uma plataforma estreita (e.g., delta do Congo). Durante esse processo de aporte junto ao talude, após o início do escoamento numa região subaérea, a corrente hiperpicnal atingiu o nível de água e começou a escoar junto ao fundo do talude no canal subaquoso previamente formado, fazendo uma analogia aos cânions e canais submarinos encontrados nas regiões de talude e planícies abissais das bacias oceânicas. 0 canal previamente formado causou uma aceleração do fluxo através do princípio da conservação de massa, modificando também seu comportamento hidrodinâmico e geométrico até que o final desse canal subaquoso chegasse ao fim e uma nova condição de equilíbrio foi reestabelecida. Através das imagens obtidas não é possível analisar se o comportamento do fluxo dentro do canal permaneceu em condição de aceleração, porém, foi possível verificar, através das imagens, tabelas e gráficos anteriormente apresentados, que a velocidade do fluxo confinado, ao sair do canal, igualou-se com o fluxo não confinado, permanecendo deste modo durante o restante do tempo do experimento.

Nos ambientes naturais (e.g., as bacias oceânicas), esse tipo de fenômeno descrito acima, pode ocorrer não 
só nos instantes iniciais do fluxo, mas sim ao longo de toda a sua duração. A aceleração instantânea do fluxo (waxing flow - Kneller, 1995) caracterizada pelos ciclos de aceleração e desaceleração devido à dinâmica interna do fluxo e da sua interface, se soma com a aceleração espacial (accumulative flow - Kneller, 1995) ao longo dessa região canalizada. Esses dois efeitos somados, indicam um grande potencial para a ocorrência de uma região onde predomine processos erosivos do fluxo hiperpicnal (comum em correntes de turbidez), ao invés de uma zona de deposição. Infelizmente, a escala reduzida de simulação utilizada neste caso, impediu que os fenômenos de erosão de sedimentos (fortemente influenciada pela tensão de cisalhamento do escoamento junto ao fundo) fossem simulados propriamente, uma vez que não foram geradas tensões de cisalhamento suficiente elevadas para remover as partículas previamente depositadas junto ao fundo. Middleton (1967) já destacou esse problema quando afirmou que a quantificação dos processos envolvendo erosão, transporte e deposição de sedimentos, nos modelos físicos, sempre apresenta uma série de dificuldades relacionadas ao entendimento e à quantificação da interação entre os sistemas: fluido e transporte de sedimento, dificultando uma completa semelhança entre os processos reais e simulados.

Outro aspecto relacionado a esse aumento de velocidade nas correntes confinadas diz respeito à capacidade de transporte e à competência do escoamento no transporte de sedimentos ao longo do escoamento. Em ambos os casos, é esperado que o acréscimo de velocidades e aceleração nessas regiões confinadas aumente a capacidade de transporte da corrente, uma vez que os grãos podem manter-se em suspensão devido ao aumento da turbulência interna e também, que a competência do fluxo aumente, uma vez que o fluxo ainda estaria confinado (como na condição de escoamento fluvial) podendo manter a energia suficiente para carregar esses grãos maiores.

As mudanças verificadas na geometria do fluxo podem indicar mudanças no transporte de sedimentos em ambientes naturais. Tradicionalmente, diz-se que a cabeça é uma zona de grande intensidade turbulenta (Simpson, 1972; Simpson \& Britter, 1979; Middleton, 1993) e que possui uma dinâmica peculiar. Neste trabalho, ao longo do tempo que a corrente confinada foi formada, notou-se que a cabeça da corrente se destacava em relação ao corpo, indicando que, no ambiente natural, esse tipo de comportamento da corrente possa causar processos erosivos localizados, garantindo assim que o escoamento e transporte de sedimentos do fluxo alcance regiões mais distais da bacia.

\section{Conclusão}

O experimento demonstrou que o confinamento de um fluxo hiperpicnal, juntamente com a declividade imposta a ele, causa uma modificação no seu padrão de escoamento (aceleração no tempo e espaço), gerando diferentes comportamentos hidrodinâmicos, geométricos e deposicionais. Em ambientes naturais, esse tipo de fenômeno localizado e curto pode ocorrer por longas distâncias, com uma duração maior. Este confinamento com duração longa poderia proporcionar um aumento na velocidade do fluxo, propiciando a ocorrência maior de processos erosivos em relação a processos deposicionais.

A modelagem física apresentada mostrou-se uma ferramenta eficiente para estudar um fenômeno complexo, como as correntes hiperpicnais, sob uma condição de confinamento, analisando suas características, tanto espaciais quanto de aceleração, por um curto período de tempo. Mesmo com a análise baseada somente em imagens, foi possível avaliar e comparar o comportamento de um fluxo confinado e um não confinado ao longo de seu desenvolvimento.

Agradecimentos - Os autores agradecem a Petróleo Brasileiro S.A., pelo incentivo à pesquisa nacional. A todos os pesquisadores e funcionários do Necod que participaram desse projeto de pesquisa, desde a parte de construção do canal à análise e interpretação dos dados. Agradecemos, também, aos revisores Antônio Cosme Del Rey e Giorgio Basilici pelas sugestões e críticas construtivas ao texto.

\section{Referências}

Baptista, M.B. \& Coelho, M.M.L.P. 2003. Fundamento de Engenharia Hidráulica. 2 ed. rev., Belo Horizonte: Editora UFMG. 440 p.

Bates, C.C. 1953. Rational theory of delta formation. AAPG Bulletin, 37: 2119-2162.

Boffo, C.H. 2010. Caracterização física de correntes de densidade em ambientes salinos sob diferentes contrastes de densidades. Porto Alegre, 117p. Dissertação de Mestrado, Programa de Pós-graduação em Recursos Hídricos e Saneamento Ambiental, Instituto de Pesquisas Hidráulicas, Universidade Federal do Rio Grande do Sul.

Ducker, R.E. 2007. Avaliação da capacidade portante de frações finas nas correntes de turbidez. Porto Alegre, 85p. Dissertação de Mestrado. Programa de Pós-graduação em Recursos Hídricos e Saneamento Ambiental, Instituto de Pesquisas Hidráulicas, Universidade Federal do Rio Grande do Sul.

Del Rey, A.C. 2006. Simulação física de processos gravitacionais subaquosos: uma aproximação para o entendimento da sedimentação marinha profunda. Porto Alegre, 229p. Tese de Doutorado, Programa de Pós-graduação em Geociências, Instituto de Geociências, Universidade Federal do Rio Grande do Sul.

Fabian, S. 2002. Modelagem física de correntes de densidade em canal bidimensional de declividade variável. Porto Alegre, 130p. Dissertação de Mestrado, Instituto de Pesquisas Hidráulicas, Universidade Federal do Rio Grande do Sul.

Felix, M. \& Peakall, J. 2006. Transformation of debris flows into turbidity currents: mechanisms inferred from laboratory experiments. Sedimentology, 53: 107-123.

Gladstone, C. \& Pritchard, D. 2010. Patterns of deposition from experimental turbidity currents with reversing 
buoyancy. Sedimentology, 57: 53-84.

Kneller, B. 1995. Beyond the turbidite paradigm: physical models for deposition of turbidites and their implications for reservoir prediction. In: Hartley, A.J. \& Prosser, D.J. (Eds.) Characterization of deep marine clastic systems. London, The Geological Society Special Publication, p. 3149.

Lamb, M.P., McElroy, B., Kopriva, B., Shaw, J. \& Mohrig, D. 2010. Linking river-flood dynamics to hyperpycnal-plume deposits: experiments, theory, and geological implications. Geological Society of America Bulletin, 122: 1389-1400.

Manica, R. 2002. Modelagem física de correntes de densidade não conservativas em canal tridimensional de geometria simplificada. Porto Alegre, 161p. Dissertação de Mestrado, Programa de Pós-graduação em Recursos Hídricos e Saneamento Ambiental, Universidade Federal do Rio Grande do Sul.

Manica, R. 2009. Geração de correntes de turbidez de alta densidade: condicionantes hidráulicos e deposicionais. Porto Alegre, 424p. Tese de Doutorado, Programa de Pós-graduação em Recursos Hídricos e Saneamento Ambiental, Universidade do Rio Grande do Sul.

Manica, R. 2012. Sediment Gravity Flows: Study Based on Experimental Simulations. In: Schulz H.E. Simões, A.L.A \& Lobosco, R.L. Hydrodynamics - Natural Water Bodies. In: E Harry Edmar Schulz, André Luiz Andrade Simões and Raquel Jahara Lobosco. (Org.). Hydrodynamics - Natural Water Bodies. Hydrodynamics - Natural Water Bodies. Rijeka: InTech - Open Access Publisher, 2012, v. 1, p. 263286.

Middleton, G.V. 1967. Experiments on density and turbidity currents III. Deposition of sediment: Canadian Journal of

Manuscrito 497

Editores: Iran S. Correa e Maria do Carmo Lima e Cunha.
Earth Sciences, 4: 475 - 505.

Middleton, G.V. 1993. Sediment deposition from turbidity currents. Annual Review of Earth Planet Science, 21: 89114.

Middleton, G.V. \& Hampton, M.A. 1976. Subaqueous sediment transport and deposition by sediment gravity flows. In: Stanley, D.J. \& Swift, D.J.P (Eds.) Marine Sediment Transport and Environmental Management. New York, John Wiley, p. 197-218.

Mulder, T. \& Syvitski, J.P.M. 1995. Turbidity currents generated at river mouths during exceptional discharges to the world oceans. Journal of Geology, 103: 285-299.

Mulder, T. \& Alexander, J. 2001. The physical character of subaqueous sedimentary density flows and their deposits. Sedimentology, 48: 269-299.

Mulder, T., Syvitski, J.P.M., Migeon, S., Faugères, J.C. \& Savoyed, B., 2003. Marine hyperpycnal flows: initiation, behavior and related deposits. A review. Marine and Petroleum Geology, 20: 861-882.

Simpson, E.J. 1972. Effects of the lower boundary on the head of a gravity current. Journal of Fluid Mechanics, 53: 759-768.

Simpson, E.J. \& Britter, R.E. 1979. The dynamics of the head of a gravity current advancing over a horizontal surface. Journal of Fluid Mechanics, 94: 447-495.

Stow, D.A.V. 1994. Deep sea processes of sediment transport and deposition. In: Pye, K. 1. Ed. Sediment Transport and Depositional Process. Oxford, Blackwell Science, p. 257293.

Wheatcroft, R.A., 2000. Oceanic flood sedimentation: a new perspective. Continental Shelf Research, 20: 2059-2066. 